\title{
MicroRNA-3666-induced suppression of SIRT7 inhibits the growth of non-small cell lung cancer cells
}

\author{
HONGYANG SHI ${ }^{1}$, YUQIANG JI ${ }^{2}$, DEXIN ZHANG $^{1}$, YUN LIU $^{1}$ and PING FANG ${ }^{1}$ \\ ${ }^{1}$ Department of Respiratory Medicine, The Second Affiliated Hospital of Xi'an Jiaotong University, \\ Xi'an, Shaanxi 710004; ${ }^{2}$ Department of Cardiovascular Disease, \\ Xi'an No.1 Hospital, Xi'an, Shaanxi 710002, P.R. China
}

Received April 19, 2016; Accepted August 17, 2016

DOI: $10.3892 /$ or.2016.5063

\begin{abstract}
Sirtuin7 (SIRT7) plays an important role in many cancer types, but its function in non-small cell lung cancer (NSCLC) remains unclear. This study investigated the biological role and underlying mechanism of SIRT7 in NSCLC. Results showed that SIRT7 was highly expressed in NSCLC cell lines, as detected by real-time quantitative polymerase chain reaction and western blot analysis. SIRT7 knockdown by small interfering RNA (siRNA) significantly inhibited the growth of NSCLC cells and induced their apoptosis. Bioinformatics algorithms indicated that SIRT7 was a putative target of microRNA-3666 (miR-3666). Dual-luciferase reporter assay demonstrated that miR-3666 could target the 3'-untranslated region of SIRT7. Western blot analysis revealed that miR-3666 could regulate the protein expression of SIRT7. The miR-3666 overexpression significantly inhibited NSCLC cell growth. The restoration of SIRT7 protein expression significantly abrogated the effect of the miR-3666 overexpression. Moreover, SIRT7 depletion induced by siRNA or miR-3666 overexpression promoted the expression of proapoptotic genes. Taken together, our study suggests that SIRT7 functions as an oncogene in NSCLC, and miR-3666 can target SIRT7 to inhibit NSCLC cell growth by promoting the proapoptotic signaling pathway. Thus, this study provides novel insights into the development of new and potential treatments for NSCLC.
\end{abstract}

Correspondence to: Dr Hongyang Shi, Department of Respiratory Medicine, The Second Affiliated Hospital of Xi'an Jiaotong University, 157 West 5th Road, Xi'an, Shaanxi 710004, P.R. China E-mail: shihongyanghy@163.com

Abbreviations: SIRTs, sirtuins; NSCLC, non-small cell lung cancer; RT-qPCR, real-time quantitative polymerase chain reaction; siRNA, small interfering RNA; UTR, untranslated region; mRNA, messenger RNA; miRNAs, microRNAs; miR-3666, microRNA-3666

Key words: SIRT7, non-small cell lung cancer, cell growth, miRNAs

\section{Introduction}

Lung cancer is a leading cause of cancer-related deaths worldwide, and $>80 \%$ of lung cancers are diagnosed as non-small cell lung cancer (NSCLC) (1). Unfortunately, the morbidity and mortality rates of lung cancer in China have remained high and are even continuously increasing; as a consequence, this disease has resulted in heavy economic burden $(2,3)$. Despite the advancements in treatment strategies, the overall 5 -year survival rate of lung cancer remains low (1). Cancer treatment remains challenging because of the malignant growth and distant metastasis of cancer cells (4). However, underlying mechanisms remain largely unknown. Therefore, innovative research efforts should be performed to understand the mechanism of carcinogenesis and to develop new and effective therapeutic methods.

Sirtuins (SIRTs) are nicotinamide adenine dinucleotide oxidized form-dependent deacetylases belonging to class III histone deacetylase (5). SIRTs are also called longevity proteins because of their distinct roles in extending lifespan (5). SIRTs regulate various biological processes, including stress responses, DNA repair, inflammation, metabolism, apoptosis, cell cycle, and senescence; they also play critical roles in cardiovascular and neurodegenerative diseases and cancer (5-8). Thus far, seven members of the SIRT family (SIRT1-7) have been characterized in mammals (9). Among the SIRTs, SIRT1 is the most widely explored; this SIRT is implicated in various cellular and metabolic processes (10). The important role of SIRT2 has been observed in neurodegenerative diseases (11). SIRT3 is another thoroughly investigated SIRT that represents a major mitochondrial deacetylase $(12,13)$. SIRT4 and SIRT5 participate in metabolic processes $(14,15)$. SIRT6 possibly regulates DNA damage and genome integrity $(16,17)$. SIRT7 is the latest characterized SIRT and its function study has just begun (18). SIRT7 exhibits nuclear localization and regulates ribosomal RNA synthesis $(19,20)$. SIRT7 has been implicated in various cellular processes, such as cellular survival, protein synthesis, chromatin remodeling, and lipid metabolism (18). SIRT7 has also been proposed as an oncogene in various cancer types, including hepatocellular carcinoma (21) and colorectal cancer (22). Nevertheless, the role of SIRT7 in lung cancer remains unclear. 
MicroRNAs (miRNAs), a group of small and non-coding RNAs consisting of $\sim 22$ nucleotides, have been extensively investigated in terms of cancer pathogenesis and treatments because of their negative regulatory effect on gene expression (23). miRNAs can directly target the 3'-untranslated region (UTR) of target messenger RNA (mRNA) via complementary sequences; as a result, mRNA becomes unstable and undergoes degradation; thus, translation is inhibited (24-26). Therefore, miRNAs participate in various biological processes, such as cell proliferation, apoptosis, migration, and invasion (24). A series of miRNAs has been aberrantly expressed in cancer tissues, such as NSCLC, functioning as oncogenes or tumor suppressive genes $(27,28)$. These miRNAs participate in cancer pathogenesis and thus can be potentially used as new diagnostic biomarkers and therapeutic targets $(27,28)$.

In our study, to better understand the biological role of SIRT7 in NSCLC, we analyzed the expression of SIRT7 in several NSCLC cell lines. The results showed that SIRT7 was highly expressed in NSCLC cell lines. SIRT7 knockdown by small interfering RNA (siRNA) significantly inhibited the growth of NSCLC cells and promoted their apoptosis. To develop a novel inhibitor for SIRT7, we predicted novel and potential miRNAs that could target and regulate SIRT7 through bioinformatics algorithms. A novel miRNA, the miR-3666, contained the predicted binding sites for 3'-UTR of SIRT7. The finding was confirmed through dual-luciferase reporter assay. Further data revealed that miR-3666 markedly inhibited the protein expression of SIRT7. The overexpression of miR-3666 significantly inhibited NSCLC cell growth directly through SIRT7. Moreover, the loss of SIRT7 induced by siRNA or miR-3666 activated the proapoptotic gene expression. Thus, miR-3666/SIRT7 could be used as a novel and potential molecular target for NSCLC treatment.

\section{Materials and methods}

Cell lines. Normal human keratinocyte cell line (HaCaT), human NSCLC cell lines (A549, H1299, NCI-H157, and SK-MES-1), and 293T cells were purchased from American Type Culture Collection (ATCC, Manassas, VA, USA). These cells were grown in Dulbecco's modified Eagle's medium (DMEM; Gibco, Rockville, MD, USA) supplemented with $10 \%$ fetal calf serum (Gibco) and penicillin $(100 \mathrm{U} / \mathrm{ml}) / \mathrm{strep}-$ tomycin $(100 \mu \mathrm{g} / \mathrm{ml})$ and then placed in a humidified incubator containing $5 \% \mathrm{CO}_{2}$ at $37^{\circ} \mathrm{C}$.

Real-time quantitative polymerase chain reaction ( $R T-q P C R)$. Total RNAs were isolated using an RNeasy kit and an miRNeasy mini kit (Qiagen, Dusseldorf, Germany) according to the manufacturer's instructions. cDNA was synthesized with M-MLV reverse transcriptase (Clontech, Palo Alto, CA, USA) and a Primescript RT reagent kit (Takara, Dalian, China). PCR amplification was performed using a SYBR Premix Ex Taq GC kit (Takara) on an ABI7500 real-time PCR detection system (Applied Biosystems, Carlsbad, CA, USA). Glyceraldehyde-3-phosphate dehydrogenase (GAPDH) or U6 was also used as an internal control. Fold changes relative to the control were calculated via the $2^{-\Delta \Delta C t}$ method.
Western blot analysis. Protein extractions were separated through $12.5 \%$ sodium dodecyl sulfate polyacrylamide gel electrophoresis and subsequently transferred to a polyvinylidene difluoride membrane (Millipore, Boston, MA, USA). The membrane was blocked with $3.5 \%$ non-fat milk and probed with primary antibodies at $4^{\circ} \mathrm{C}$ overnight. Afterward, the membrane was incubated with horseradish peroxidase-conjugated secondary antibodies $(1: 2,000$, Santa Cruz Biotechnology, Santa Cruz, CA, USA) for $1 \mathrm{~h}$. Protein bands were developed through chemiluminescence (Amersham Biosciences, Little Chalfont, UK). Band intensity was quantified using Image-Pro Plus 6.0 (Media Cybernetics, Inc., Rockville, MD, USA). The primary antibodies (anti-SIRT7 and anti-GAPDH) used in this study were purchased from Santa Cruz Biotechnology.

Cell transfection. SIRT7 siRNA (siSIRT7-1:5'-CCUGCCGUG UGAGGCGGAA-3' and siSIRT7-2: 5'-GCCGUGUGAGGCG GAAGCG-3') and control siRNA (siControl: 5'-CCUUGCGU GGGAGCGCGAA-3') were synthesized by Invitrogen (Carlsbad, CA, USA). miR-3666 mimics and negative control miRNA mimics (NC miRNA) were purchased from OriGene Technologies (Beijing, China). For SIRT7 overexpression, the open reading frame of SIRT7 without 3'-UTR was cloned into pcDNA3.1 plasmid (BioVector, Beijing, China). The siRNA, miRNA mimics, or plasmids were transfected by using Lipofectamine $^{\mathrm{TM}} 2000$ (Invitrogen) according to the manufacturer's instructions.

3-(4,5-Dimethyl-thiazol-2-yl)-2,5-diphenyltetrazolium bromide (MTT) assay. Cell growth was detected through an MTT assay. In brief, the cells were seeded into 96-well plates at a density of $1 \times 10^{4}$ cells/well and cultured overnight. After transfection was performed for $48 \mathrm{~h}, 20 \mu \mathrm{l}$ of MTT stock solution (Sigma, St. Louis, MO, USA) was added to each well and incubated for $4 \mathrm{~h}$; afterward, $200 \mu \mathrm{l}$ of dimethyl sulfoxide/well (Sigma) was added. Absorbance was detected at $490 \mathrm{~nm}$ with a microplate spectrophotometer (Bio-Tek Instruments, Winooski, VT, USA).

Caspase-3 activity assay. Cell apoptosis was detected through caspase-3 activity assay by using a commercial kit (Roche Life Science, Shanghai, China). The cells were lysed in ice-cold cell lysis buffer. The supernatant was collected, and protein concentration was measured. Subsequently, $100 \mu \mathrm{g}$ of protein was incubated with $50 \mu 1$ of reaction buffer and $5 \mu \mathrm{l}$ of $4 \mathrm{mM}$ DEVD-pNA substrate at $37^{\circ} \mathrm{C}$ for $2 \mathrm{~h}$. Absorbance was determined at $405 \mathrm{~nm}$ by using a microplate spectrophotometer (Bio-Tek Instruments).

Dual-luciferase reporter assay. The 3'-UTR of SIRT7 containing the predicted binding site or a mutant at miR-3666 binding site was cloned into pmirGLO dual-luciferase vector (Promega, Madison, WI, USA) downstream of the luciferase gene. The recombinant dual-luciferase vector was co-transfected with miR-3666 mimics into 293T cells by using Lipofectamine ${ }^{\mathrm{TM}}$ 2000 (Invitrogen). After $48 \mathrm{~h}$ of incubation, luciferase activity was determined by using a dual-Glo luciferase assay system (Promega).

Statistical analysis. Data were expressed as mean \pm standard deviation. Statistical analyses were performed with Student's 

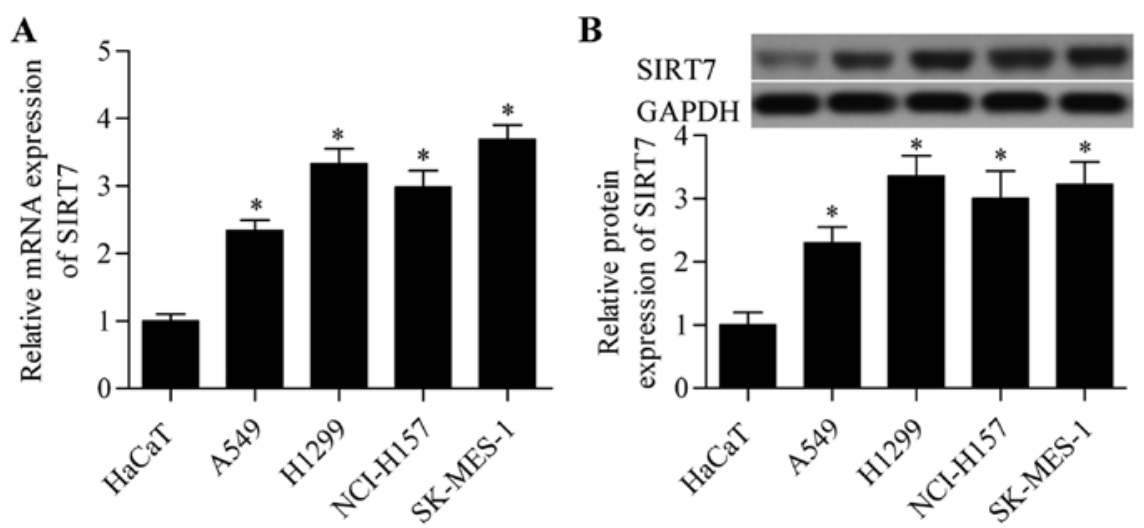

Figure 1. SIRT7 is overexpressed in NSCLC cells. The mRNA (A) and protein (B) expression levels of SIRT7 were detected in A549, H1299, NCI-H157, and SK-MES-1 cells through real-time quantitative polymerase chain reaction (RT-qPCR) or western blot analysis, respectively. Normal human keratinocyte $\mathrm{HaCaT}$ cells were used as the control cells. "p<0.05 vs. HaCaT.
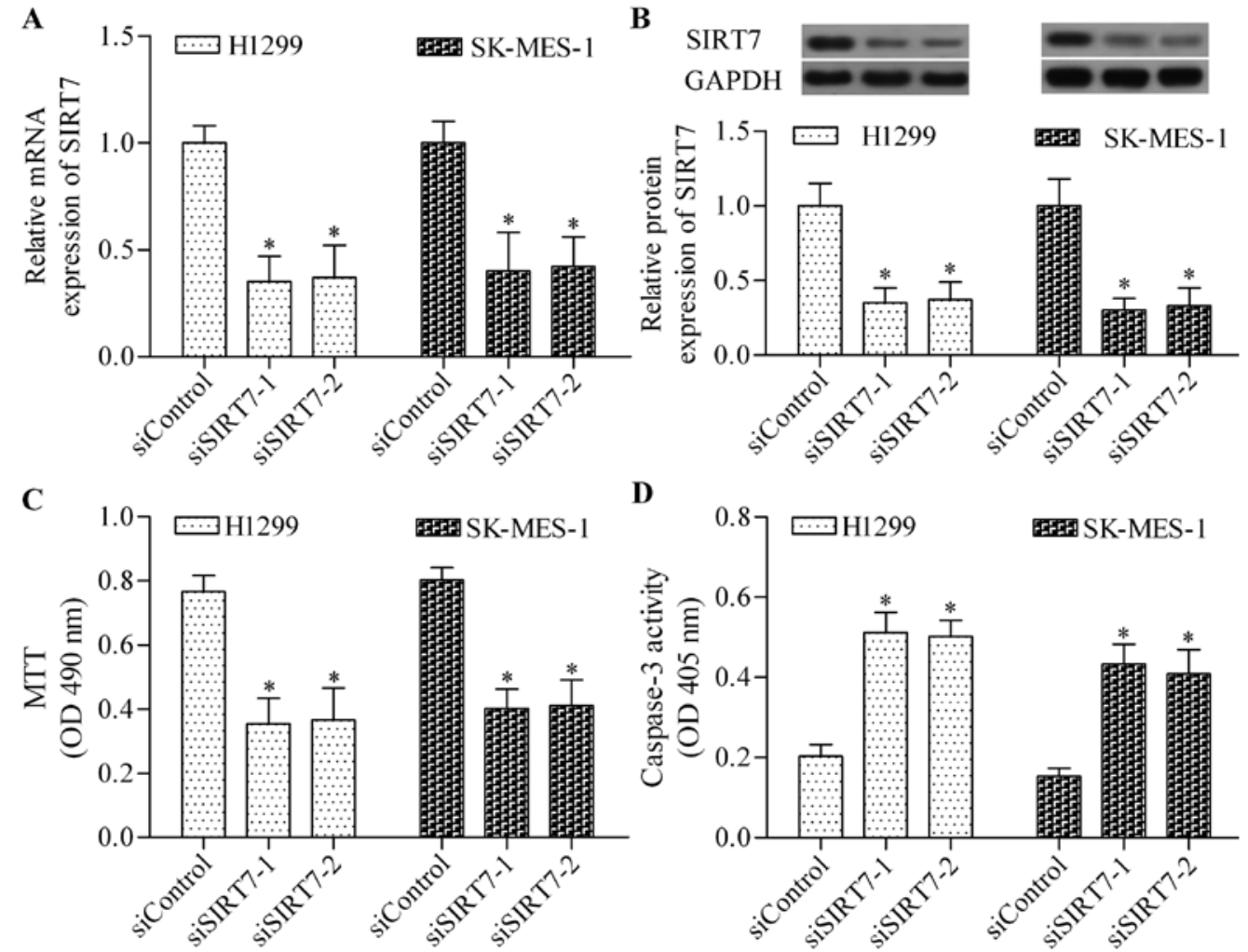

Figure 2. SIRT7 knockdown by siRNA inhibites NSCLC cell growth. Cells were transfected with siSIRT7-1 or siSIRT7-2 for 48 h. The efficiency of siSIRT7-1 and siSIRT7-2 on SIRT7 mRNA (A) and protein (B) expression in H1299 and SK-MES-1 cells was detected by RT-qPCR and western blot analysis, respectively. (C) The effect of SIRT7 depletion on cell growth was detected by MTT assay. (D) The effect of SIRT7 depletion on cell apoptosis was detected by caspase- 3 activity assay. ${ }^{*} \mathrm{p}<0.05$ vs. siControl.

t-test or ANOVA with a Bonferroni correction in SPSS version 11.5 software (SPSS Inc., Chicago, IL, USA). A p-value of $<0.05$ was considered statistically significant.

\section{Results}

SIRT7 is highly expressed in NSCLC cells. To investigate the potential role of SIRT7 in NSCLC, we detected the expression of SIRT7 in several NSCLC cell lines. RT-qPCR analysis revealed that the mRNA expression level of SIRT7 in NSCLC cell lines was significantly higher than that in $\mathrm{HaCaT}$ cells
(Fig. 1A). The protein expression level of SIRT7 in NSCLC cells was also higher than that in the control $\mathrm{HaCaT}$ cells (Fig. 1B). These results suggested that SIRT7 plays an important role in NSCLC.

SIRT7 knockdown inhibits the cell growth of NSCLC cells. To investigate the biological role of SIRT7 in NSCLC, we performed a loss-of-function experiment through cell transfection by using siSIRT7. The expression of SIRT7 in H1299 and SK-MES-1 cells was significantly depleted after transfection was conducted with siSIRT7-1 and siSIRT7-2 (Fig. 2A and B). 


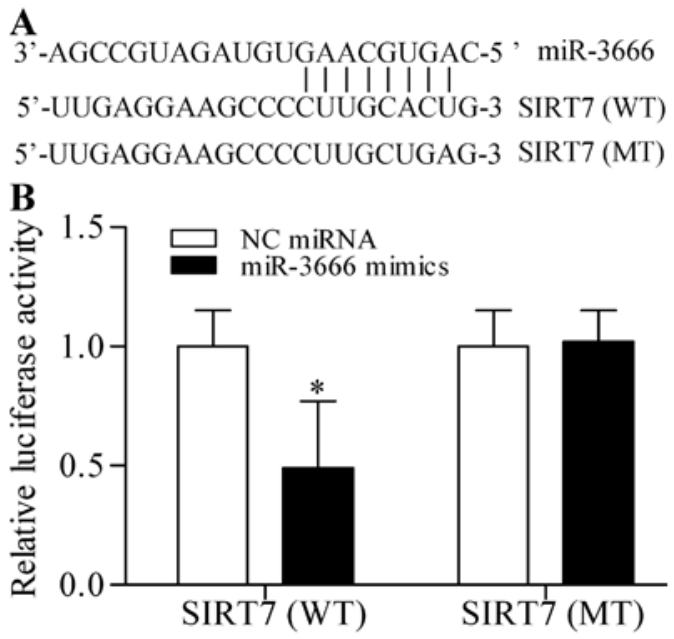

Figure 3. miR-3666 targets the 3'-UTR of SIRT7. (A) The sequences of miR3666 and its putative target sites in the 3'-UTR of SIRT7. (B) SIRT7 3'-UTR dual-luciferase reporter assay. The wild-type (WT) or mutant type (MT) reporter vectors and miR-3666 mimics were cotransfected into $293 \mathrm{~T}$ cells for $48 \mathrm{~h}$. ${ }^{\mathrm{p}} \mathrm{p}<0.05 \mathrm{vs}$. negative control (NC) miRNA.

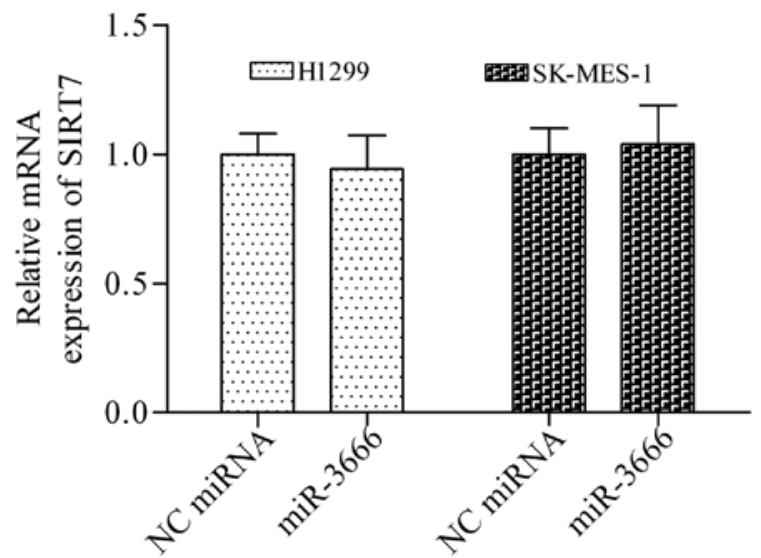

Figure 4. Effect of miR-3666 on SIRT7 mRNA expression. Cells were transfected with miR-3666 mimics for $48 \mathrm{~h}$. The effect of miR-3666 mimics on SIRT7 mRNA expression in H1299 and SK-MES-1 cells was detected by RT-qPCR analysis.
Subsequently, the effect of SIRT7 depletion on the cell growth of NSCLC cells was detected. The results showed that SIRT7 knockdown significantly inhibited the growth of H1299 and SK-MES-1 cells, as detected by the MTT assay (Fig. 2C). Furthermore, SIRT7 knockdown also markedly increased the caspase-3 activity, thereby implying a high level of apoptosis induced (Fig. 2D). Taken together, the data indicated that SIRT7 had an oncogenic role in NSCLC.

miR-3666 targets and inhibits SIRT7 protein expression. Considering the important role of SIRT7 in NSCLC, we may assume that targeting SIRT7 is a promising strategy to prevent NSCLC cancer. miRNAs are novel tools used to treat lung cancer by negatively regulating oncogene or tumor suppressive gene $(28,29)$. We also predicted the novel and potential miRNAs that could target and regulate SIRT7 through bioinformatics algorithms. Interestingly, miR-3666, a novel tumor suppressive miRNA, contained a theoretical seed region to target the 3'-UTR of SIRT7 (Fig. 3A). To confirm this prediction, we cloned the 3'-UTR of SIRT7 (wild-type, WT) into pmirGLO dual-luciferase reporter vector and co-transfected miR-3666 mimics with this vector in 293T cells. Luciferase activities were quantified in the transfected cells and the results showed that miR-3666 mimics significantly decreased the luciferase activity (Fig. 3B). By contrast, miR-3666 mimics did not evidently affect the mutant 3'-UTR constructs (mutant type, MT) (Fig. 3B). To investigate whether miR-3666 targeting the mRNA of SIRT7 regulates the expression of SIRT7, we detected the effect of miR-3666 mimics on SIRT7 expression in NSCLC cells. miR-3666 mimics did not significantly affect the mRNA of SIRT7 (Fig. 4). By contrast, miR-3666 mimics markedly decreased the protein expression of SIRT7 (Fig. 6A and B). Therefore, miR-3666 regulates the protein expression of SIRT7.

miR-3666 inhibits NSCLC cell growth through targeting SIRT7. Considering the regulatory effect of miR-3666 on SIRT7 expression, we speculated that miR-3666 could inhibit NSCLC cell growth. To test this hypothesis, we transfected NSCLC cells with miR-3666 mimics and detected its effect on
A

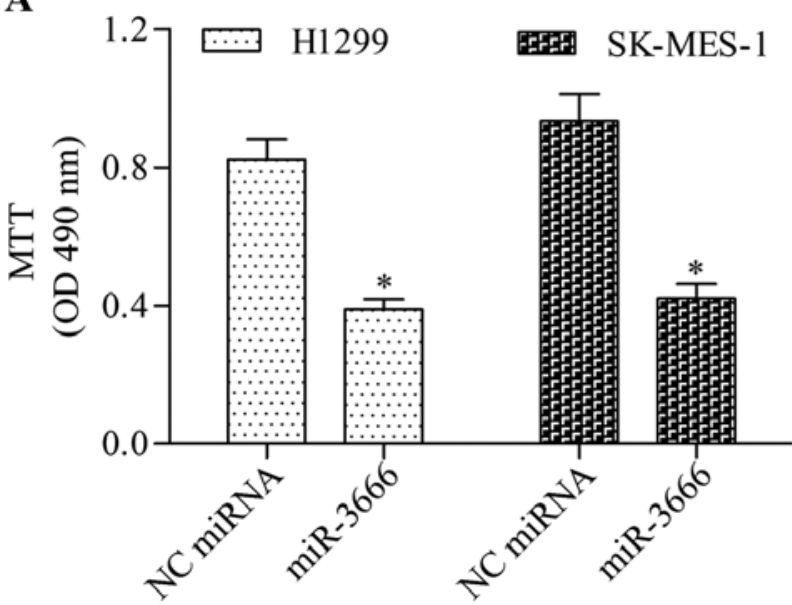

B

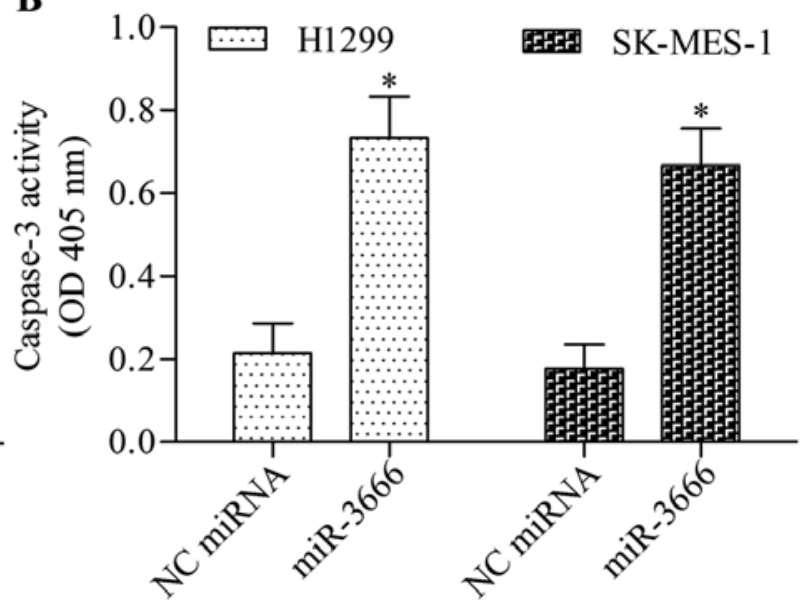

Figure 5. Effect of miR-3666 on NSCLC cell growth. Cells were transfected with miR-3666 mimics for $48 \mathrm{~h}$. The effect of miR-3666 mimics on cell growth (A) and cell apoptosis (B) was detected by MTT assay and caspase- 3 activity assay, respectively. ${ }^{*}$ p $<0.05$ vs. NC miRNA. 
$\mathbf{A}$

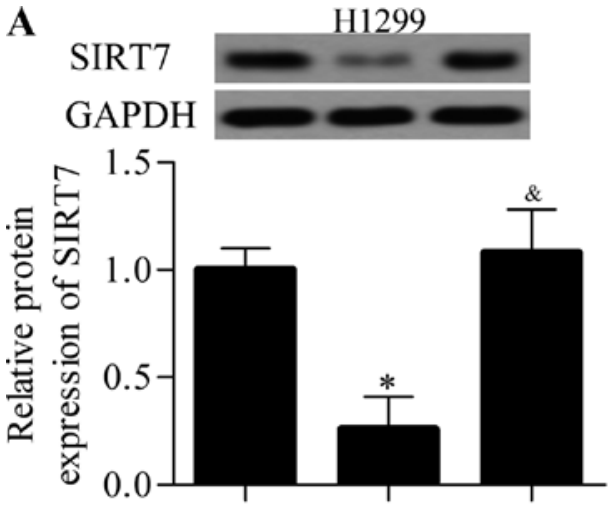

C

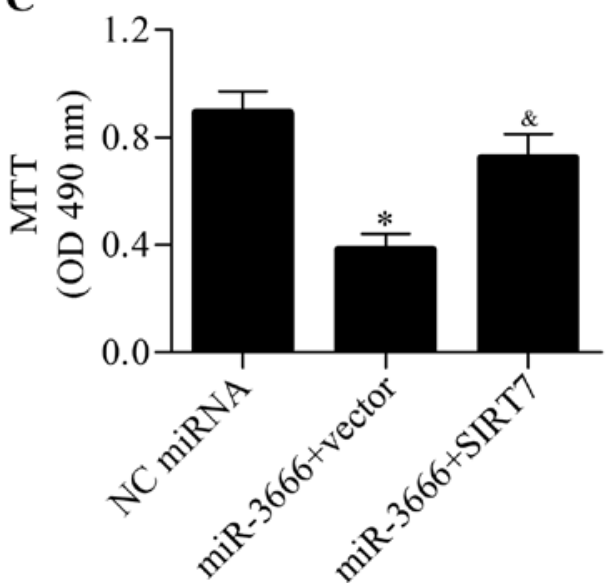

B

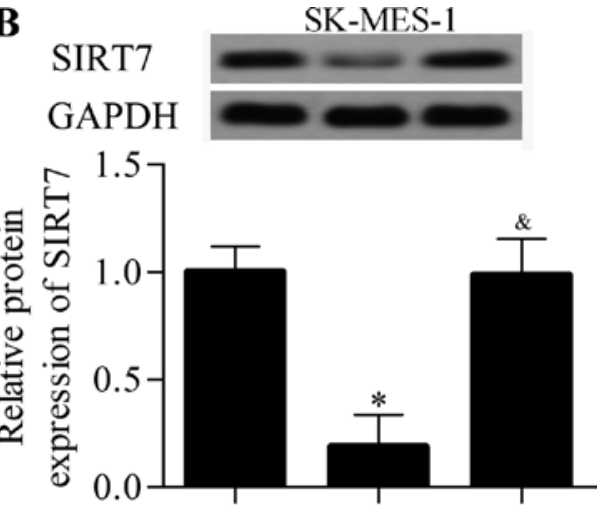

D

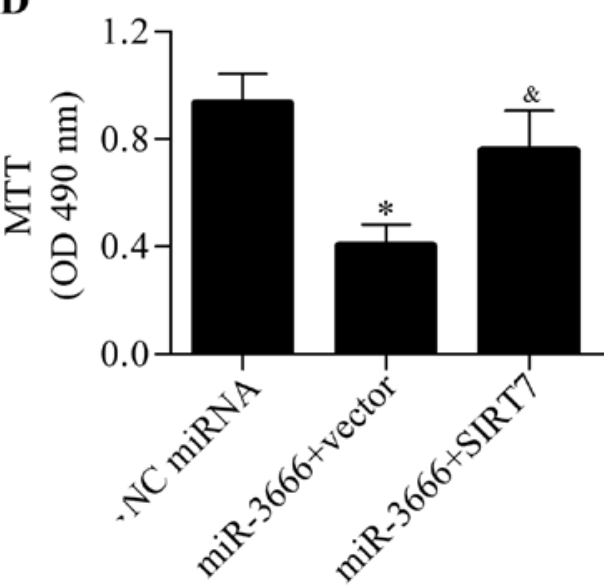

Figure 6. SIRT7 overexpression reverses the effect of miR-3666. Cells were cotransfected with miR-3666 mimics and pcDNA3.1-SIRT7 expressing vector for $48 \mathrm{~h}$. The protein expression of SIRT7 in H1299 (A) and SK-MES-1 (B) cells was detected by western blot analysis. The cell growth was detected by MTT assay in H1299 (C) and SK-MES-1 (D) cells. "p<0.05 vs. NC miRNA; ${ }^{*}$ p $<0.05$ vs. miR-3666+vector.
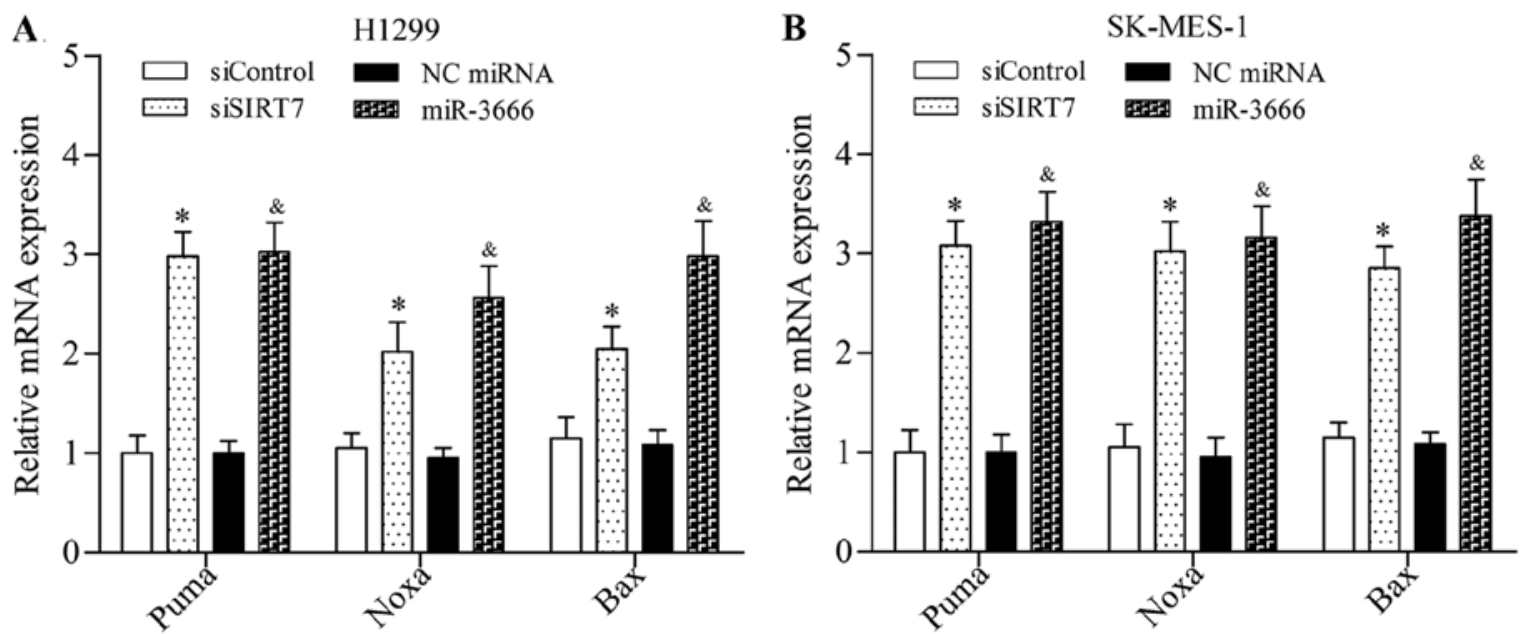

Figure 7. Loss of SIRT7 promotes the pro-apoptotic gene expression. Cells were transfected with SIRT7 siRNA or miR-3666 mimics for $48 \mathrm{~h}$. The mRNA expression levels of Puma, Noxa, and Bax in transfected H1299 (A) and SK-MES-1 (B) cells were detected by RT-qPCR analysis. "p<0.05 vs. siControl; ${ }^{\&} \mathrm{p}<0.05$ vs. NC miRNA.

cell growth and apoptosis. The results showed that miR-3666 overexpression significantly inhibited the growth (Fig. 5A) and apoptosis (Fig. 5B) of NSCLC cells. To validate whether miR-3666 inhibited NSCLC cell growth through SIRT7, we performed a rescue experiment. The cells were cotransfected with miR-3666 mimics and SIRT7-overexpressing vector (without 3'-UTR). The results showed that the decreased protein expression of SIRT7 induced by miR-3666 mimics was significantly restored by the transfection of SIRT7overexpressing vector (Fig. 6A and B). Furthermore, restoring SIRT7 expression markedly decreased the inhibitory effect of miR-3666 on NSCLC cell growth (Fig. 6C and D). These 
data indicated that miR-3666 inhibited NSCLC cell growth through targeting SIRT7.

Loss of SIRT7 promotes the activation of pro-apoptotic gene expression. To further elucidate the underlying mechanism of SIRT7 in regulating NSCLC cell growth and apoptosis, we detected the effect of the loss of SIRT7 on pro-apoptotic gene expression. The results showed that SIRT7 depletion induced by siRNA or miR-3666 mimics significantly increased the mRNA expression levels of Puma, Noxa, and Bax in NSLCLC cells (Fig. 7). Taken together, these results suggested that SIRT7 inhibition promoted the activation of pro-apoptotic signaling pathway.

\section{Discussion}

In this study, we demonstrated a crucial role of SIRT7 in NSCLC. SIRT7 was overexpressed in NSCLC cell lines, and SIRT7 knockdown by siRNA significantly inhibited cell growth and induced cell apoptosis of NSCLC. Intriguingly, miR-3666, a novel tumor suppressive miRNA (30,31), could target and inhibit SIRT7 protein expression through directly interacting with the 3'-UTR of SIRT7 mRNA. The overexpression of miR-3666 significantly inhibited NSCLC cell growth through inhibiting SIRT7. Our data indicated an important role of miR-3666/SIRT7 in regulating NSCLC cell growth.

SIRT7 regulates the ribosomal RNA synthesis $(19,20)$. SIRT7 is highly expressed in highly proliferative tissues; SIRT7 is implicated in cell growth regulation (9). This protein also plays a critical role in cancers (18). Barber et al reported that SIRT7 is essential for the maintenance of cancerous phenotype because SIRT7 contributes to the maintenance of H3K18 deacetylation leading to the suppression of many tumor suppressor genes (32). The depletion of SIRT7 in cancer cells reduces the anchorage-independent growth and cell proliferation (32). SIRT7 is highly expressed in hepatocellular carcinoma tissues, and its knockdown results in cell cycle arrest and inhibits cell grow in vitro and in vivo (21). SIRT7 is also gradually increased during cervical cancer progression (33). In colorectal cancer, SIRT7 protein expression is significantly correlated with tumor stage, lymph node metastasis, and poor patient survival; SIRT7 knockdown also suppresses the motility, proliferation, and colony formation of colorectal cancer cells via the mitogen-activated protein kinase pathway (22). Furthermore, SIRT7 is upregulated in gastric cancer and is correlated with disease stage, metastasis, and survival (34). SIRT7 knockdown also inhibits gastric cancer cell growth in vitro and in vivo (34). In addition, SIRT7 is highly expressed in ovarian cancer cell lines, and its loss reduces cell growth and colony formation and promotes cell apoptosis of ovarian cancer cells (35). High SIRT7 expression is also found in breast cancer and is correlated with high histological grade and poor survival rate $(36,37)$. These findings indicate that SIRT7 functions as an oncogene. In line with these findings, our results revealed that this protein functioned as an oncogene in lung cancer. Our results further demonstrated that SIRT7 was overexpressed in NSCLC cell lines, and the knockdown of this gene impeded NSCLC cell growth. On the contrary, SIRT7 possesses tumor-suppressor properties (38). McGlynn et al demonstrated that decreased SIRT7 expression is associ- ated with an aggressive tumor phenotype and poor survival (38). SIRT7 knockdown also induces multidrug resistance in breast cancer cells (39).

miRNAs represent novel inhibitors for gene expression (24-26). SIRT7 can be regulated by certain miRNAs $(21,40)$. Kim et al reported that tumor suppressive miRNAs, miR-125a-5p, and miR-125b can target and inhibit SIRT7 to suppress the growth of hepatocellular carcinoma cells (21). Zhao and Wang found that miR-125b inhibits the proliferation of hepatocellular carcinoma cells by targeting SIRT7 (40). Another study reports that miR-125b also inhibits bladder cancer development by targeting and inhibiting SIRT7 (41). A recent study revealed that SIRT7 is also a target gene of miR-93, which regulates adiposity (42). In our study, we found that the miR-3666 could directly target and inhibit SIRT7 expression. miR-3666 has been suggested as an oncogene in cervical cancer and can inhibit cancer cell invasion and metastases by targeting zinc finger E-box binding homeobox 1 (30). Low miR-3666 expression is detected in thyroid carcinoma, but the miR-3666 overexpression significantly inhibits the growth of thyroid carcinoma cancer cells by targeting the met proto-oncogene (31). In this study, we found that miR-3666 also functioned as a potential tumor suppressor in NSCLC. The miR-3666 overexpression could inhibit NSCLC cell growth by suppressing SIRT7. Our study further documented that SIRT7 could be targeted and inhibited by specific miRNAs. Therefore, miR-3666 could be used as a novel inhibitor of SIRT7 to inhibit SIRT7-related cancers, such as NSCLC.

SIRT7 plays an important role in regulating cell apoptosis (43). The depletion of SIRT7 triggers apoptosis in mammalian cells (19). SIRT7 can also prevent cardiomyocyte apoptosis through deacetylation of p53 and inhibition of cell apoptosis activation (43). Moreover, SIRT7 knockdown increases proapoptotic proteins and inhibits anti-apoptotic proteins in gastric cancer cells (34). In osteosarcoma cells, SIRT7 induces resistance to doxorubicin-induced apoptosis by inhibiting the activation of the pro-apoptotic signaling pathway (44). In line with these findings, our results demonstrated that the loss of SIRT7 significantly promoted the expression of pro-apoptotic genes, including Puma, Noxa, and Bax. Our results further confirmed the role of SIRT7 in regulating cell apoptosis in cancer cells.

Our results indicated that SIRT7 functioned as an oncogene in NSCLC. miR-3666-induced SIRT7 inhibition could suppress cell growth and trigger apoptosis of NSCLC cells. Therefore, miR-3666/SIRT7 may be considered novel and potential molecular targets for NSCLC treatment.

\section{Acknowledgements}

This study was supported by the Fundamental Research Funds for the Central Universities (no. XJJ2012057) and Natural Science Basic Research Project of Shaanxi Province (no. 2016JQ8054).

\section{References}

1. Siegel RL, Miller KD and Jemal A: Cancer statistics, 2015. CA Cancer J Clin 65: 5-29, 2015. 
2. Qiu ZX, Sun RF, Mo XM and Li WM: The p70S6K specific inhibitor PF-4708671 impedes non-small cell lung cancer growth. PLoS One 11: e0147185, 2016.

3. Zhang C, Zhang H, Shi J, Wang D, Zhang X, Yang J, Zhai Q and Ma A: Trial-based cost-utility analysis of icotinib versus gefitinib as second-line therapy for advanced non-small cell lung cancer in China. PLoS One 11: e0151846, 2016.

4. Steeg PS: Metastasis suppressors alter the signal transduction of cancer cells. Nat Rev Cancer 3: 55-63, 2003.

5. Houtkooper RH, Pirinen E and Auwerx J: Sirtuins as regulators of metabolism and healthspan. Nat Rev Mol Cell Biol 13: 225-238, 2012

6. Bosch-Presegué $\mathrm{L}$ and Vaquero A: The dual role of sirtuins in cancer. Genes Cancer 2: 648-662, 2011.

7. North BJ and Verdin E: Sirtuins: Sir2-related NAD-dependent protein deacetylases. Genome Biol 5: 224, 2004.

8. Matsushima S and Sadoshima J: The role of sirtuins in cardiac disease. Am J Physiol Heart Circ Physiol 309: H1375-H1389, 2015.

9. Michishita E, Park JY, Burneskis JM, Barrett JC and Horikawa I: Evolutionarily conserved and nonconserved cellular localizations and functions of human SIRT proteins. Mol Biol Cell 16: 4623-4635, 2005.

10. Meng X, Tan J, Li M, Song S, Miao Y and Zhang Q: Sirt1: Role under the condition of ischemia/hypoxia. Cell Mol Neurobiol: Mar 14, 2016 (Epub ahead of print).

11. Donmez G and Outeiro TF: SIRT1 and SIRT2: Emerging targets in neurodegeneration. EMBO Mol Med 5: 344-352, 2013.

12. Lombard DB, Alt FW, Cheng HL, Bunkenborg J, Streeper RS, Mostoslavsky R, Kim J, Yancopoulos G, Valenzuela D, Murphy A, et al: Mammalian Sir2 homolog SIRT3 regulates global mitochondrial lysine acetylation. Mol Cell Biol 27: 8807-8814, 2007.

13. Onyango P, Celic I, McCaffery JM, Boeke JD and Feinberg AP: SIRT3, a human SIR2 homologue, is an NAD-dependent deacetylase localized to mitochondria. Proc Natl Acad Sci USA 99: 13653-13658, 2002.

14. Nakagawa T, Lomb DJ, Haigis MC and Guarente L: SIRT5 Deacetylates carbamoyl phosphate synthetase 1 and regulates the urea cycle. Cell 137: 560-570, 2009.

15. Haigis MC, Mostoslavsky R, Haigis KM, Fahie K, Christodoulou DC, Murphy AJ, Valenzuela DM, Yancopoulos GD Karow M, Blander G, et al: SIRT4 inhibits glutamate dehydrogenase and opposes the effects of calorie restriction in pancreatic beta cells. Cell 126: 941-954, 2006.

16. Mao Z, Hine C, Tian X, Van Meter M, Au M, Vaidya A, Seluanov A and Gorbunova V: SIRT6 promotes DNA repair under stress by activating PARP1. Science 332: 1443-1446, 2011.

17. McCord RA, Michishita E, Hong T, Berber E, Boxer LD, Kusumoto R, Guan S, Shi X, Gozani O, Burlingame AL, et al: SIRT6 stabilizes DNA-dependent protein kinase at chromatin for DNA double-strand break repair. Aging (Albany, NY) 1: 109-121, 2009.

18. Kiran S, Anwar T, Kiran M and Ramakrishna G: Sirtuin 7 in cell proliferation, stress and disease: Rise of the seventh sirtuin! Cell Signal 27: 673-682, 2015.

19. Ford E, Voit R, Liszt G, Magin C, Grummt I and Guarente L: Mammalian Sir2 homolog SIRT7 is an activator of RNA polymerase I transcription. Genes Dev 20: 1075-1080, 2006.

20. Grob A, Roussel P, Wright JE, McStay B, Hernandez-Verdun D and Sirri V: Involvement of SIRT7 in resumption of rDNA transcription at the exit from mitosis. J Cell Sci 122: 489-498, 2009.

21. Kim JK, Noh JH, Jung KH, Eun JW, Bae HJ, Kim MG, Chang YG, Shen Q, Park WS, Lee JY, et al: Sirtuin7 oncogenic potential in human hepatocellular carcinoma and its regulation by the tumor suppressors MiR-125a-5p and MiR-125b. Hepatology 57: 1055-1067, 2013.

22. Yu H, Ye W, Wu J, Meng X, Liu RY, Ying X, Zhou Y, Wang H, Pan $C$ and Huang W: Overexpression of sirt7 exhibits oncogenic property and serves as a prognostic factor in colorectal cancer. Clin Cancer Res 20: 3434-3445, 2014.

23. Ebrahimi A and Sadroddiny E: MicroRNAs in lung diseases: Recent findings and their pathophysiological implications. Pulm Pharmacol Ther 34: 55-63, 2015.
24. Mendell JT and Olson EN: MicroRNAs in stress signaling and human disease. Cell 148: 1172-1187, 2012.

25. Bartel DP: MicroRNAs: Genomics, biogenesis, mechanism, and function. Cell 116: 281-297, 2004.

26. Winter J, Jung S, Keller S, Gregory RI and Diederichs S: Many roads to maturity: microRNA biogenesis pathways and their regulation. Nat Cell Biol 11: 228-234, 2009.

27. Cortinovis D, Monica V, Pietrantonio F, Ceresoli GL, La Spina CM and Wannesson L: MicroRNAs in non-small cell lung cancer: Current status and future therapeutic promises. Curr Pharm Des 20: 3982-3990, 2014

28. Feng B, Zhang K, Wang R and Chen L: Non-small-cell lung cancer and miRNAs: Novel biomarkers and promising tools for treatment. Clin Sci (Lond) 128: 619-634, 2015.

29. Barger JF and Nana-Sinkam SP: MicroRNA as tools and therapeutics in lung cancer. Respir Med 109: 803-812, 2015.

30. Li L, Han LY, Yu M, Zhou Q, Xu JC and Li P: Pituitary tumortransforming gene 1 enhances metastases of cervical cancer cells through miR-3666-regulated ZEB1. Tumour Biol: 2015 Sep 17, 2015 (Epub ahead of print).

31. Wang G, Cai C and Chen L: MicroRNA-3666 regulates thyroid carcinoma cell proliferation via MET. Cell Physiol Biochem 38: 1030-1039, 2016

32. Barber MF, Michishita-Kioi E, Xi Y, Tasselli L, Kioi M, Moqtaderi Z, Tennen RI, Paredes S, Young NL, Chen K, et al: SIRT7 links H3K18 deacetylation to maintenance of oncogenic transformation. Nature 487: 114-118, 2012.

33. Singh S, Kumar PU, Thakur S, Kiran S, Sen B, Sharma S, Rao VV, Poongothai AR and Ramakrishna G: Expression/localization patterns of sirtuins (SIRT1, SIRT2, and SIRT7) during progression of cervical cancer and effects of sirtuin inhibitors on growth of cervical cancer cells. Tumour Biol 36: 6159-6171, 2015.

34. Zhang S, Chen P, Huang Z, Hu X, Chen M, Hu S, Hu Y and Cai T: Sirt7 promotes gastric cancer growth and inhibits apoptosis by epigenetically inhibiting miR-34a. Sci Rep 5: 9787, 2015.

35. Wang HL, Lu RQ, Xie SH, Zheng H, Wen XM, Gao X and Guo L: SIRT7 exhibits oncogenic potential in human ovarian cancer cells. Asian Pac J Cancer Prev 16: 3573-3577, 2015.

36. Aljada A, Saleh AM, Alkathiri M, Shamsa HB, Al-Bawab A and Nasr A: Altered sirtuin 7 expression is associated with early stage breast cancer. Breast Cancer (Auckl) 9: 3-8, 2015.

37. Geng Q, Peng H, Chen F, Luo R and Li R: High expression of Sirt7 served as a predictor of adverse outcome in breast cancer. Int J Clin Exp Pathol 8: 1938-1945, 2015.

38. McGlynn LM, McCluney S, Jamieson NB, Thomson J, MacDonald AI, Oien K, Dickson EJ, Carter CR, McKay CJ and Shiels PG: SIRT3 \& SIRT7: Potential novel biomarkers for determining outcome in pancreatic cancer patients. PLoS One 10: $0131344,2015$.

39. Aljada A, Saleh AM and Al Suwaidan S: Modulation of insulin/IGFs pathways by sirtuin-7 inhibition in drug-induced chemoreistance. Diagn Pathol 9: 94, 2014.

40. Zhao L and Wang W: miR-125b suppresses the proliferation of hepatocellular carcinoma cells by targeting Sirtuin7. Int J Clin Exp Med 8: 18469-18475, 2015.

41. Han Y, Liu Y, Zhang H, Wang T, Diao R, Jiang Z, Gui Y and Cai Z: Hsa-miR-125b suppresses bladder cancer development by down-regulating oncogene SIRT7 and oncogenic long noncoding RNA MALAT1. FEBS Lett 587: 3875-3882, 2013

42. Cioffi M, Vallespinos-Serrano M, Trabulo SM, FernandezMarcos PJ, Firment AN, Vazquez BN, Vieira CR, Mulero F, Camara JA, Cronin UP, et al: MiR-93 controls adiposity via inhibition of Sirt7 and Tbx3. Cell Rep 12: 1594-1605, 2015.

43. Vakhrusheva O, Smolka C, Gajawada P, Kostin S, Boettger T, Kubin T, Braun T and Bober E: Sirt7 increases stress resistance of cardiomyocytes and prevents apoptosis and inflammatory cardiomyopathy in mice. Circ Res 102: 703-710, 2008.

44. Kiran S, Oddi V and Ramakrishna G: Sirtuin 7 promotes cellular survival following genomic stress by attenuation of DNA damage, SAPK activation and p53 response. Exp Cell Res 331: 123-141, 2015. 